\title{
A giant vagal Schwannoma: a case report
}

\begin{abstract}
We report a rare case of a giant vagal schwannoma that was presented as a slowgrowing cervical mass. Our purpose is to describe the clinical presentation, diagnostic imaging and histopathological aspects.
\end{abstract}

Keywords: vagus nerve schwannoma, cervical mass, tomography, magnetic resonance imaging

\author{
Volume 6 Issue 3 - 2019

\section{Diego Jordão Lino Dias, Vinícius Martins Valois and Sérgio Furlan \\ Department of Radiology, Heliopolis Hospital, Brazil}

Correspondence: Diego Jordão Lino Dias, Department of Radiology, Heliopolis Hospital, I 40 Imbituba Street, 185 apartment, Vila Prudente, São Paulo-SP, 03।32-090, Brazil, Tel 55 (I4)991613327, Email diegojordao@msn.com

Received: May 08, 2019 | Published: June 14, 2019

\section{Introduction}

Parapharyngeal tumors are rare. Among them, benign neurogenic tumors are the most common types, most of which are represented by vagus nerve schwannomas and corresponds to $2-5 \%$ of neurogenic tumors of the head and neck. ${ }^{1-3}$ Epidemiology demonstrates that these tumors affect mainly adults between the third and sixth decades of life, with similar involvement in both genders. ${ }^{2,4}$ Usually benign, Schwannomas are asymptomatic tumors with minimal risk of malignant transformation, slow growth with an increase ratio of 2.5 to $3 \mathrm{~mm}$ per year, tending to be diagnosed late as large, encapsulated and with poorly mobile solid masses. Despite radiological characteristics well described, the diagnosis can only be confirmed after complete resection of the tumor and its anatomopathological evaluation..$^{1-3}$ In this case report, we describe the histological and radiological aspects of a giant vagal schwannoma (GVS), extending from the base of the skull to the upper mediastinum with involvement of the recurrent laryngeal nerve.

\section{Case report}

A 28-year-old woman with a history of cervical bulging with 12 months of evolution, complaining of dyspnea which has been increased in the last 4 months, associated with weight loss, dysphagia and neck pain. Initially the patient was evaluated by the head and neck surgery team. They performed a direct laryngoscopy with a biopsy, observing a submucosal lesion that increased the vallecula bilaterally and epiglottis. This lesion blocked the visualization of the supraglottis and vocal cords. Subsequently, the patient had respiratory insufficiency and tracheostomy was required, performed imaging study with computed tomography-CT (Figure 1) and magnetic resonance imaging-MRI (Figure 2) which shows morphological aspects of the lesion. The final diagnosis of a giant vagal schwannoma was determined by the histology and immunohistochemistry analysis (Figure 3). Allowing the correct surgical propedeutic.
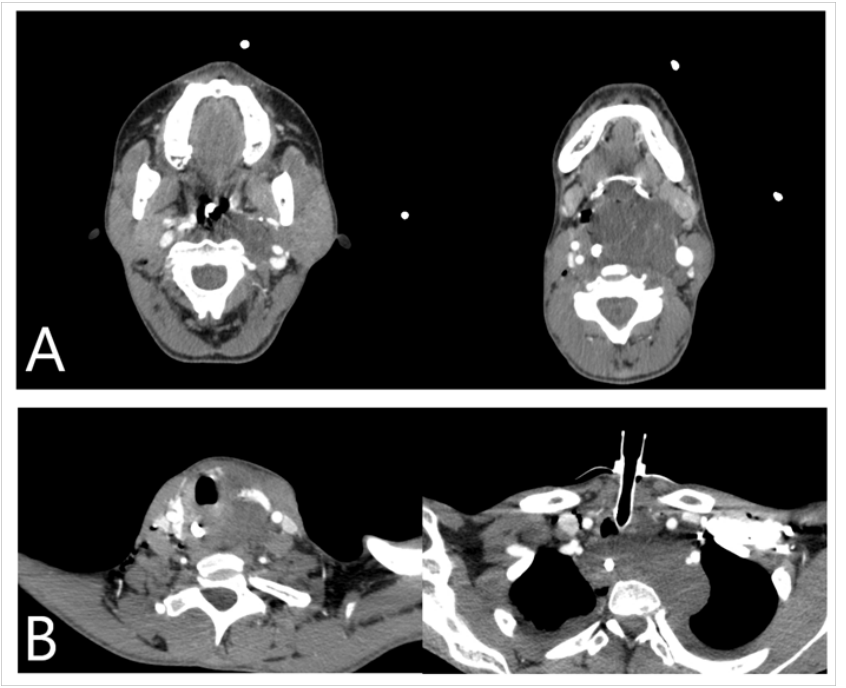

Figure I Axial CT findings:A and B Showed a hypoattenuating solid expansive lesion, affecting the left superior parapharyngeal space, carotid space, extends inferiorly to the hypopharynx and retropharyngeal space, obliterating the supraglottis and glottis. 


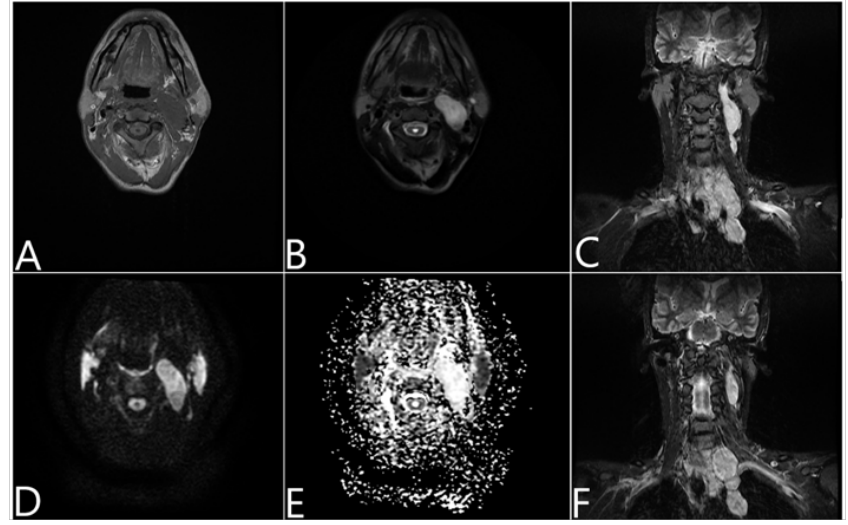

Figure 2 MRI findings: $(A)$ In the TI-weighted sequence it presented low signal. (B) In the T2-weighted sequence presented high signal. (D/E) Diffusion weighted imaging (DWI) and apparent diffusion coefficient (ADC), without evidence of diffusion restriction. (C/F) CoronalT2 STIR showing all involvement of the vagus nerve from the pars vascularis of the jugular foramen extending to the superior mediastinum and returning to the recurrent laryngeal nerve.

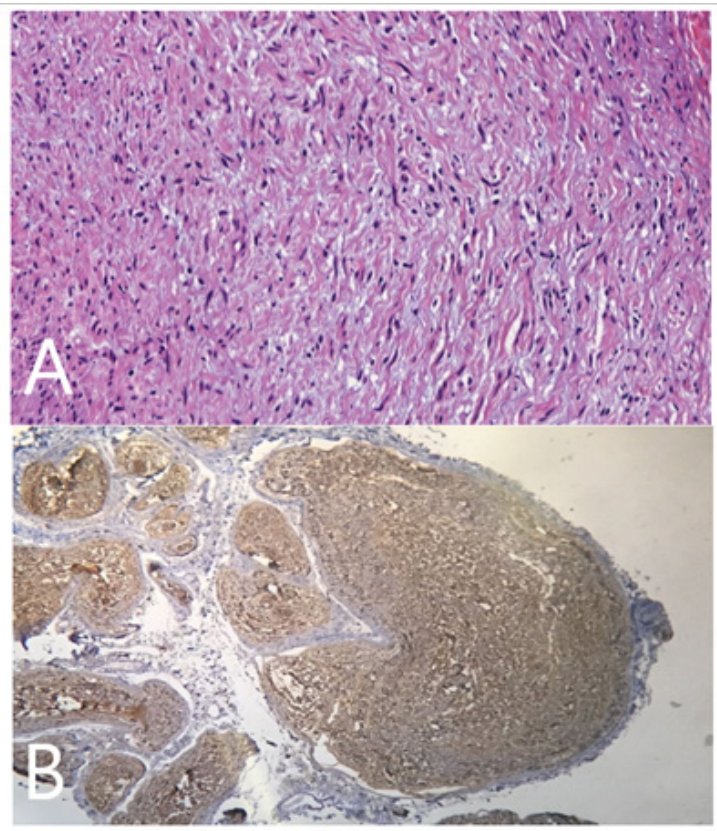

Figure 3 Histological findings: (A) Encapsulated lesion with typical benignappearing, spindle-shaped cells (H\&E, original. magn. $x$ 400). (B) SI00 protein immunohistochemical positivity of the schwannoma.

\section{Discussion and conclusion}

The first description of a parapharyngeal schwannoma case occurred in 1933 by Figi. ${ }^{5,6}$ Since then, there have been few reports of cases with giant mass presentations. In this case, GVS is an extremely rare entity, so the understanding the anatomy, epidemiology, clinical presentation, imaging, and histologic findings can make the difference. ${ }^{7}$ In anatomy, it should be emphasized that the vagus nerve runs within the carotid between the internal or common carotid artery, and the internal jugular vein as a neurovascular bundle, traveling towards the neck, chest, and abdomen. ${ }^{4,8}$ In the case presented, it is interesting to observe the involvement of the recurrent laryngeal nerve, which was determinant for the obstruction of the airways. Preoperative diagnostic exams include CT, MRI, and biopsy., $\mathrm{CT}$ is important for discarding bone lesions, delimiting anatomical spaces, and assessing the extent of the lesion. But MRI can lead to diagnostic suspicion in the preoperative period, considering the well circumscribed mass located between the internal jugular vein and the carotid artery. Not presenting anomalous enhancement or restriction to the diffusion. ${ }^{3}$ As the histological pattern, malignant schwannoma tumors differ from the benign type in their higher mitotic rate, presence of necrosis and infiltrative appearance, in addition to irregular positivity for the S-100 protein. In our case, the results were very typical. ${ }^{7}$ In short, knowledge of the anatomy and path of the vagus with its recurrences are essentials, as well as understanding the behavior of slow-growing tumors of head and neck is the key for this diagnosis.

\section{Acknowledgments}

None.

\section{Conflicts of interest}

The author declares that there is no conflict of interest.

\section{References}

1. Sreevatsa MR, Srinivasarao RV. Three cases of vagal nerve schwannoma and review of literature. Indian J Otolaryngol Head Neck Surg. 2011;63(4):310-312.

2. Ramdass AA, Yao M, Natarajan S, et al. A Rare Case of Vagus Nerve Schwannoma Presenting as a Neck Mass. Am J Case Rep. 2017; 18:908-911.

3. Vijendra SS, Rao RA, Prasad V, et al. Niprupama M. A giant vagal schwannoma with unusual extension from skull base to the mediastinum. J Can Res Ther. 2015;11:970-973.

4. Chiofalo MG, Longo F, Marone U, et al. Cervical vagal schwannoma. A case report. Acta Otorhinolaryngol Ital. 2009;29(1):33-35.

5. Noh SH, Zhang HY. Hoarseness after Section of a Cervical Vagus Nerve Schwannoma. The nerve. 2017; 3(1):18-20.

6. Figi FA. Solitary neurofibroma of the pharynx. Arch Otolaryngol. 1933; $17: 386-389$

7. Anil G, Tan TY. CT and MRI Evaluation of Nerve Sheath Tumors of the Cervical Vagus Nerve. American Journal of Roentgenology. 2011;197:195-201.

8. Langner E, Del Negro A, Akashi HK, et al. Schwannomas in the head and neck: retrospective analysis of 21 patients and review of the literature. Sao Paulo Medical Journal. 2007;125(4):220-222. 